\title{
What We Can Learn from the Light Curves of GD 358 and PG $1351+489$
}

\author{
M. H. Montgomery \\ The University of Texas at Austin, Department of Astronomy, 1 University Station, \\ C1400, Austin, TX 78712, USA \\ Delaware Asteroseismic Research Center, Mt. Cuba Observatory, Greenville, \\ DE 19807, USA
}

\begin{abstract}
We present the results of light curve fits to two DBV white dwarfs, PG 1351+489 and GD 358. For both fits, we include recent improvements in calculations relating the bolometric and observed flux variations. We provide a preliminary map of convection across the DB instability strip, and we show how this allows us to choose between two possible spectroscopic fits to PG $1351+489$.
\end{abstract}

\section{Astrophysical Context}

White dwarf stars come in two basic varieties: those with nearly pure helium surface layers (DBs) and those with nearly pure hydrogen surface layers (DAs). Each of these types contains a subtype of pulsators, denoted by DBV and DAV, respectively. In fact, the DBV GD 358 is the original member of the class of DBVs: it was discovered and predicted to pulsate by Winget (Winget 1982; Winget et al. 1982) and, as such, it is probably the most extensively studied DBV.

Since $\sim 98 \%$ of all stars become white dwarfs, white dwarfs hold the key to understanding late stages of stellar evolution. In particular, the pulsators, through their observed oscillation frequencies, provide us with detailed information about their internal structure. In this regard, GD 358 is again one of the most successful examples. Asteroseismological fits to its frequencies have yielded constraints on not just is mass, $T_{\text {eff }}$, and rotation rate, but also its internal composition and chemical profiles (Metcalfe, Nather, \& Winget 2000; Bradley \& Winget 1994; Winget et al. 1994). 
In addition to the main pulsation frequencies, these pulsators contain information which has not been traditionally exploited: the non-linearities in the light curves of medium and large amplitude pulsators. Some work has been done in this area. It was Brickhill (1992) who originally showed the convection zone should produce the strongest nonlinearities in the light curves, and this was revisited by Wu (2001), who gave an analytical basis to this approach. Further, Yeates et al. (2005) applied Wu's approach to the problem of mode identification in DAV stars. Finally, we have recently developed a technique for directly modelling these light curves which makes full use of the nonlinearities present in the pulsations (Montgomery 2005, 2007a,b). This approach removes some of the parameter degeneracies of the second-order analytical expansion of $\mathrm{Wu}$, and has so far led to mode identifications in two stars: the DAV G 29-38 and the DBV PG $1351+489$.

\section{The Light Curve Model}

We make the same set of physical assumptions as given in section 2.1 of Montgomery (2005), i.e.,

1. The flux perturbations beneath the convection zone are sinusoidal in time and have the angular dependence of a spherical harmonic.

2. The convection zone is so thin that we may locally ignore the angular variation of the nonradial pulsations, i.e., we treat the pulsations locally as if they were radial.

3. The convective turnover timescale is so short compared to the pulsation periods that the convection zone can be taken to respond "instantaneously".

4. Only flux and temperature variations are considered, i.e., the large-scale fluid motions associated with the pulsations are ignored.

Simple mixing length theories of convection predict that the thermal response timescale of the convection zone, $\tau_{C}$, should be

$$
\tau_{C} \approx \tau_{0}\left(\frac{T_{\text {eff }}}{T_{0}}\right)^{-N}
$$

where $T_{\text {eff }}$ is the instantaneous effective temperature, $T_{0}$ is its time average, and $\tau_{0}$ is the average value of $\tau_{C}$. The exponent $N$ has a value of $\sim 90$ for the DAVs and $\sim 25$ for the DBVs. This high power of $T_{\text {eff }}$ means that other 
nonlinear processes may well be negligible in comparison. These assumptions lead to the following equation relating the fluxes:

$$
F_{\mathrm{ph}}=F_{\text {base }}+\tau_{C} \frac{d F_{\mathrm{ph}}}{d t},
$$

where $F_{\text {base }}$ is the flux incident at the base of the convection zone, $F_{\mathrm{ph}}$ is the flux which emerges from the top of the convection zone in the photosphere, and $\tau_{C}$ is the instantaneous thermal response timescale, which is a function of $T_{\text {eff }}$ and therefore $F_{\text {ph }}$.

We have also made some important technical improvements to the light curve fitting code. First, we have extended it to the case where many modes having different $\ell$ and $m$ values are simultaneously present, i.e., the flux at the base of the convection zone is now given by a sum over the number of modes:

$$
\frac{\delta F_{\text {base }}}{F_{\text {base }}}=\operatorname{Re}\left\{\sum_{j=1}^{M} A_{j} e^{i \omega_{j} t+\phi_{j}} Y_{\ell_{j} m_{j}}(\theta, \phi)\right\} .
$$

In this formula, $A_{j}, \omega_{j}, \phi_{j}, \ell_{j}$, and $m_{j}$ are the amplitude, angular frequency, phase, $\ell$, and $m$ values of the $j$-th mode, and the total number of modes is $M$.

Second, we have adapted the code to simultaneously fit an arbitrary number of observations ("runs"). This was a necessary step for applying it to the multiple runs obtained during an observing campaign. Since memory is allocated and deallocated as needed, the code typically uses only 8 MB of RAM, independent of the number of runs being fit.

Finally, we have improved the way in which we calculate the "flux correction" which is needed to convert bolometric flux variations into the variations obtained in the observed passband. Specifically, if we denote by $F_{X}$ the flux in the passband $X$, we need an estimate of the quantity $\alpha_{X}$ defined by

$$
\frac{\delta F_{X}}{F_{X}}=\alpha_{X} \frac{\delta F_{\text {bol }}}{F_{\text {bol }}},
$$

where $F_{\text {bol }}$ is the bolometric flux, and $\delta F$ is the variation in the flux due to the pulsations. Clearly, this factor depends on the wavelength coverage of the passband. Assuming an average wavelength response of $\sim 5000 \AA$, in previous analyses I estimated that $\alpha \sim 0.42$ for DBVs and $\alpha \sim 0.66$ for DAVs. These values are not that different from what one obtains by assuming a blackbody law at temperatures appropriate for these objects. For the DBVs, however, these values are significantly different from what one expects from detailed model atmospheres.

In order to better calculate $\alpha_{X}$, I employ grids of photometric indices tabulated by P. Bergeron ${ }^{1}$ (for a detailed description see Bergeron, Wesemael, \&

\footnotetext{
${ }^{1}$ http://www.astro.umontreal.ca/ bergeron/CoolingModels/
} 


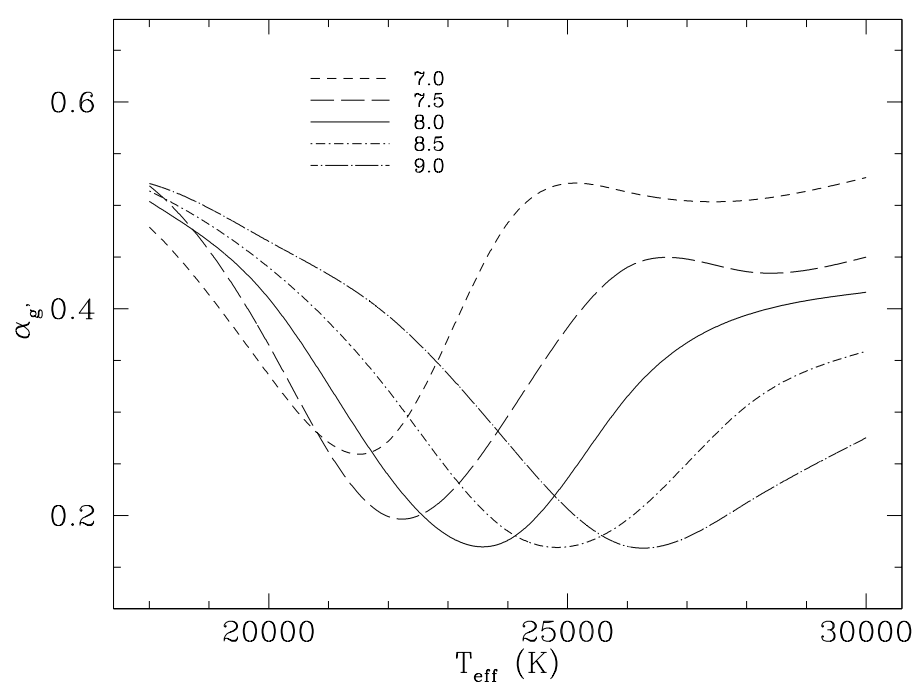

Figure 1: The flux factor $\alpha_{g^{\prime}}$ as a function of $T_{\text {eff }}$. The legend shows which values of $\log g$ correspond to the different curves.

Beauchamp 1995; Holberg \& Bergeron 2006). These tables provide absolute fluxes in different passbands (e.g., Johnson $\mathrm{U}, \mathrm{B}$, and $\mathrm{V}$ ) as a function of $T_{\text {eff }}$ and $\log g$. Since flux changes are due almost entirely to temperature variations, we can rewrite equation 4 as

$$
\begin{aligned}
\alpha_{X} & =\frac{d \ln F_{X}}{d \ln F_{\mathrm{bol}}}=\frac{1}{4} \frac{d \ln F_{X}}{d \ln T_{\mathrm{eff}}}=-\frac{\ln 10}{10} \frac{d M_{X}}{d \ln T_{\mathrm{eff}}} \\
& \approx-0.230 \frac{d M_{X}}{d \ln T_{\mathrm{eff}}},
\end{aligned}
$$

where $M_{X}$ is the absolute magnitude in the passband $X$. Our recent CCD observations have been made using a BG40 filter having an average wavelength response just below $5000 \AA$, which makes it a good match for the SDSS g' filter. Thus, we take $X$ to be $\mathrm{g}^{\prime}$ for the CCD data. Phototubes are more blue sensitive, with an average response of $\sim 4200 \AA$, which is roughly equivalent to the Johnson B filter. Thus, for data taken with phototubes we use $X=\mathrm{B}$.

In Figure 1 we show $\alpha_{g^{\prime}}$ as a function of $T_{\text {eff }}$, for several different values of $\log g$. For $\log g=8.0$ we clearly see that $\alpha_{g^{\prime}}$ is typically less than the value of 0.42 assumed in our previous analyses, and can be up to twice as small depending on the temperature range. This figure also makes clear that taking $\alpha_{g^{\prime}}$ to be a constant is not necessarily adequate for temperature excursions of several thousand degrees. Nevertheless, we defer an examination of this 
effect to a future publication and we simply assume that $\alpha_{g^{\prime}}$ is constant during the pulsations.

\section{The Fits}

Previous to the 2006 WET run on GD 358, nonlinear light curve fits had only been made for mono-periodic pulsators (the DAV G 29-38 and the DBV $P G 1351+489)$. This was because 1) the data can be folded at the period of the dominant mode, producing a high S/N "light curve", and 2) the number of possible mode identifications ( $\ell$ and $m$ values) for a single mode is small enough that all possibilities can be directly explored.

\section{GD 358}

The first multi-periodic pulsator to be explored, GD 358, violates both of the these conditions. First, due to its large number of large amplitude modes, the pulse shape obtained by folding its light curve at a mode period will not be the same as the pulse shape which would be obtained in the absence of other modes (Montgomery, 2007b). Second, due to the large number of excited modes it is not possible to search for all possible $\ell$ and $m$ values for each mode. For instance, if we take it to have of order $\sim 10$ modes observed, all of which have $\ell=1$, that yields a total number of cases of $(2 \ell+1)^{10} \sim 60000$ ! Since each fit takes of order an hour, this is completely impractical using a standard desktop computing approach. However, T. Metcalfe (personal communication) points out that this problem could run in a few hours on a computer cluster having of order one thousand nodes.

Fortunately, GD 358 has been well studied, so we believe we have a good idea what the $\ell$ and $m$ values for the main pulsation modes are (Metcalfe, Nather, \& Winget 2000; Winget et al. 1994). Furthermore, from the 2006 WET run we obtained very good frequencies for these modes. Thus, since we can assume the frequencies and mode identifications to be known, we can make nonlinear light curve fits to individual observing runs within the WET campaign which have high $\mathrm{S} / \mathrm{N}$. High $\mathrm{S} / \mathrm{N}$ data is necessary since we are mainly interested in the nonlinear part of the light curve, which itself is smaller than the linear part.

The highest $\mathrm{S} / \mathrm{N}$ data taken during the campaign were obtained with the $2.7 \mathrm{~m}$ Nordic Telescope (NOT) in La Palma. In Figure 2 we show the last night of this data together with the fit (this was a simultaneous fit of the last four night's of data taken by the NOT, of which we are showing only the last night). While not perfect, the fit clearly does a good job of reproducing most of the features in the light curve. 

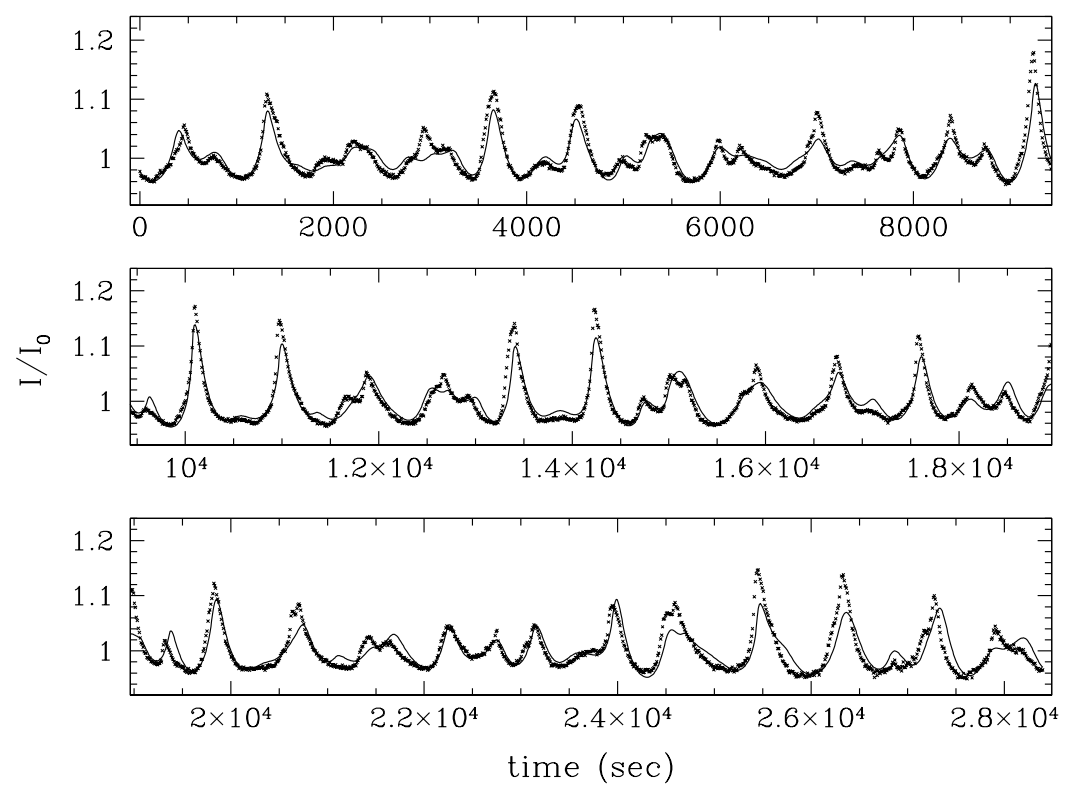

Figure 2: A light curve fit to data taken on GD 358 in the May/June 2006 WET campaign with the NOT $2.7 \mathrm{~m}$ telescope. The data points are shown as crosses and the fit is the solid curve.

Table 1: Results of 12-mode Fit for GD 358.

\begin{tabular}{|crrc|}
\hline Fit parameters: & \multicolumn{3}{c|}{$\tau_{0}=308 \mathrm{~s}, N=22.2, \theta_{\mathrm{i}}=46.6^{\circ}$} \\
\hline Period $(\mathrm{s})$ & $\ell$ & $m$ & Amplitude \\
\hline 422.561 & 1 & 1 & 0.17162 \\
423.898 & 1 & -1 & 0.13232 \\
463.376 & 1 & 1 & 0.23350 \\
464.209 & 1 & 0 & 0.10113 \\
465.034 & 1 & -1 & 0.10057 \\
571.735 & 1 & 1 & 0.25407 \\
574.162 & 1 & 0 & 0.17763 \\
575.933 & 1 & -1 & 0.32532 \\
699.681 & 1 & 0 & 0.07525 \\
810.291 & 1 & 0 & 0.35596 \\
852.502 & 1 & 0 & 0.13170 \\
962.385 & 1 & 0 & 0.12794 \\
\hline
\end{tabular}




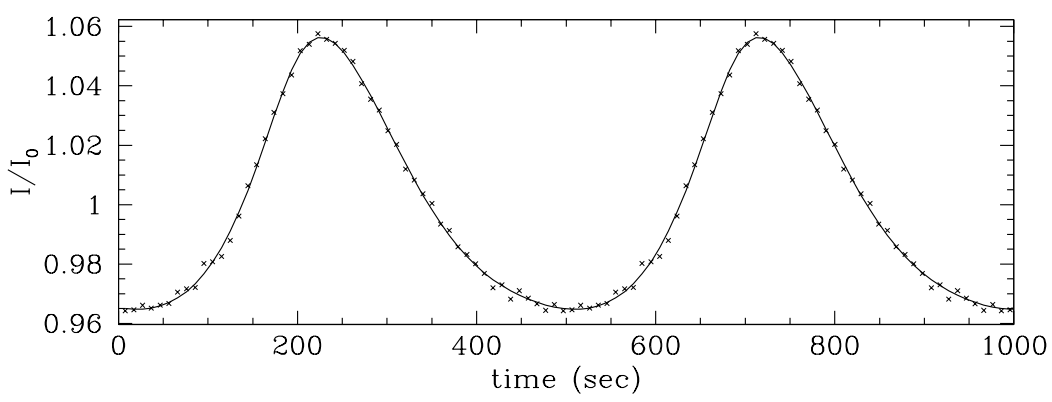

Figure 3: A light curve fit to data taken on PG $1351+489$ in May 2004 with the McDonald $2.1 \mathrm{~m}$ telescope. The data points are shown as crosses and the fit is the solid curve.

Table 2: Results of 1-mode Fit for PG $1351+489$.

\begin{tabular}{|cccccrrc|}
\hline Epoch & Period $(\mathrm{s})$ & $\ell$ & $m$ & Amplitude & $\tau_{0}(\mathrm{~s})$ & $N$ & $\theta_{\mathrm{i}}$ \\
\hline 2004 & 489.34 & 1 & 0 & 0.30989 & 89.2 & 16.0 & $58.0^{\circ}$ \\
1995 & 489.34 & 1 & 0 & 0.36529 & 86.0 & 20.5 & $56.7^{\circ}$ \\
\hline
\end{tabular}

The mode periods and identifications which we assumed are given in Table 1 , together with the amplitudes derived from the fit. In addition, this fit assumes the observations were made using the SDSS $\mathrm{g}^{\prime}$ filter, and that $T_{\text {eff }}=24900 \mathrm{~K}$ and $\log g=7.91$ for GD 358, which are the best fit zero hydrogen values of Beauchamp et al. (1999). From the calculations in the previous section, this leads us to assume a value of $\alpha_{g^{\prime}}=0.259$.

\section{PG $1351+489$}

Using the updated version of our code, we re-analyzed data taken on the nearly mono-periodic DBV PG 1351+489. The results obtained are very similar to those found earlier (Montgomery, 2005). In Figure 3 we present the fit to the folded pulse shape of the May 2004 data of the dominant 489.34 s peak. In Table 2 we give the values of the fit parameters using this data set and the data set obtained with the WET in 1995. With the exception of the amplitude, we find virtually the same values for the other parameters. 


\section{Goodness of Fit and Errors}

The fits shown in Figures 2 and 3 "look good", although this is a completely subjective, non-quantitative statement. We would obviously like to know whether these fits really have something to do with the star, and, if they do, what the uncertainties on the measured quantities $\tau_{0}, N$, and $\theta_{\mathrm{i}}$ are, for example.

For this purpose, it is tempting to apply a reduced- $\chi^{2}$ test to these data. For the NOT data on GD 358, we estimate a point-to-point scatter of about 5 mma, which leads to a reduced $\chi^{2} \sim 10$ for the light curve fit. For the folded light curve of the McDonald 2.1m data on PG $1351+489$, we estimate a scatter of $1.5 \mathrm{mma}$, which leads to a reduced $\chi^{2} \sim 0.9$. This confirms our feeling that the PG $1351+489$ fit is indeed much better than that to GD 358. In fact, the formal probability of a reduced $\chi^{2} \sim 10$ is vanishingly small. Should we therefore conclude that our fit is so poor that we do not learn anything about the star, or should we take it to mean that there are simply additional effects going on which we have not yet taken into account?

We proceed, naturally, by assuming that our fits are telling us something about the star. In an attempt to justify this belief we examine a different diagnostic: we plot how the average squared residuals decrease as fit parameters are added. First we add parameters corresponding to (linear) sinusoidal terms. Each sinusoid has an amplitude, frequency, and phase, so each adds 3 additional parameters. This is shown in Figure 4, in which the decrease in residuals out to 36 parameters is due to the addition of 12 sinusoids to the light curve fits. The right-most point in this plot corresponds to the addition of the (nonlinear) convective fit, which uses the 3 parameters $\tau_{0}, N$, and $\theta_{\mathrm{i}}$. We see that it decreases the residuals by a much larger amount than the previous terms do, so in some sense it encapsulates "more physics". This helps make the case that our fits really do capture some of the essential behavior of the star.

Error estimates are trickier, since they usually involve the assumption that the difference between the data and the fit is due to random, statistical fluctuations in the data. A glance at Figure 2 shows otherwise, however, since excursions above and below the fit tend to contain many consecutive points, not simply one or two as would be expected if the excursions were uncorrelated. This could be due to many things. First, we know that GD 358 contains other, smaller amplitude modes which we have not modelled, and these will cause smooth, coherent departures from the fit. Also, the flux correction factor, $\alpha_{g^{\prime}}$ (shown in Figure 1), is not a constant as we have assumed, and this will also cause such coherent departures. Finally, there are all the other things which the star is doing which we do not yet know about, and these are undoubtedly also having an effect. 


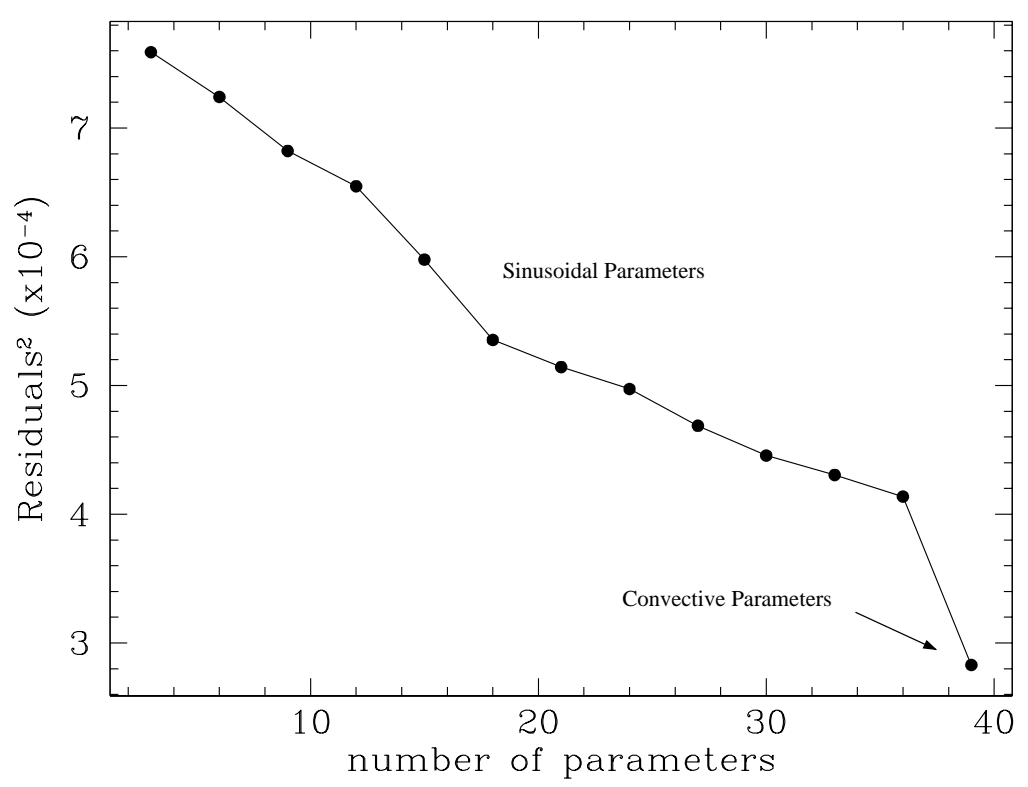

Figure 4: The squared residuals of fits to the light curve of GD 358 as a function of the number of fitted parameters. Each point corresponds to 3 parameters, and the first 12 points are the 36 parameters for the fits of 12 linear sinusoids. The last point shows the squared residuals when the convective effects are added, using the additional parameters $\tau_{0}, N$, and $\theta_{\mathrm{i}}$. The sharp downturn implies that the light curve fits do recover a significant amount of the physics in these objects, more than is obtained from the continuing addition of new frequencies to the fits.

Our current plan is to include the additional effects which we know about, i.e., the variable flux correction and the most important of the lower amplitude modes, and calculate the residuals of the fit. We will then generate synthetic, best-fit light curves to which we have added uncorrelated, Gaussian-distributed noise having the same mean value as the residuals. We will then fit this light curve and derive the convective parameters. Finally, we will repeat this process many times to derive error estimates on all quantities; this is the same procedure Montgomery (2005) used to estimate errors for the fits to G 29-38 and $P G 1351+489$. This work is currently in progress, so we defer the presentation of these results to an upcoming publication. 


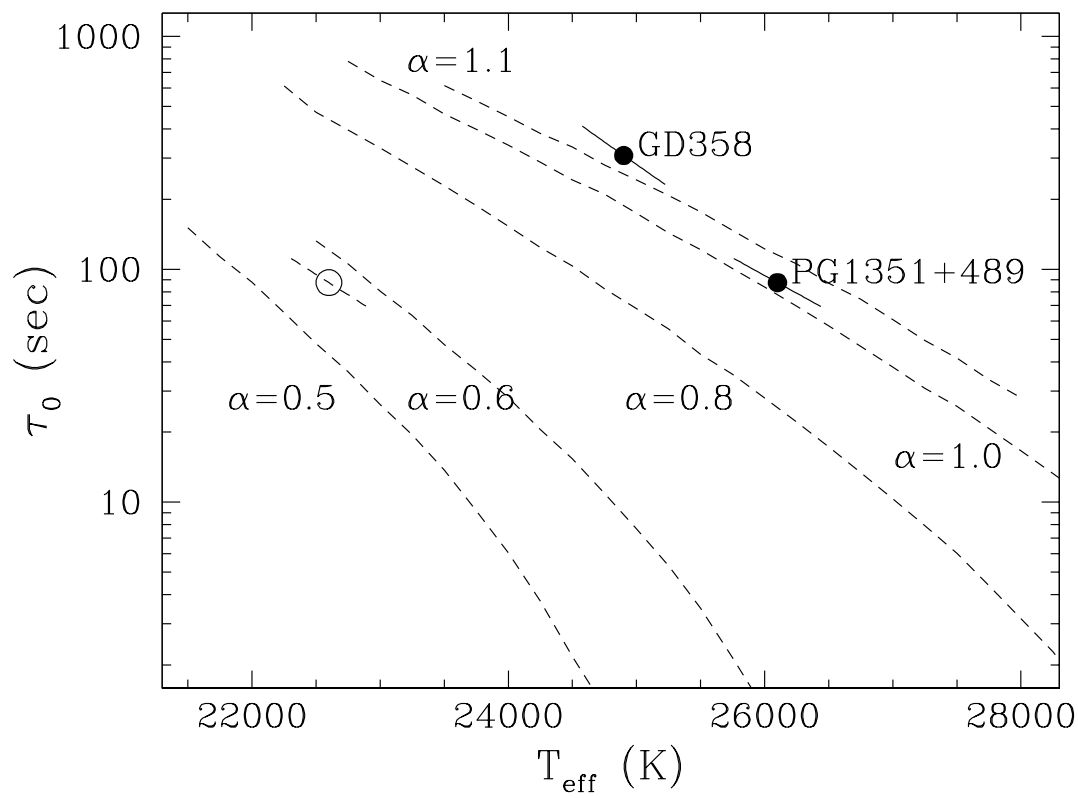

Figure 5: The convective timescale $\tau_{0}$ as a function of $T_{\text {eff }}$ across the DBV instability strip. The labelled points are from the preceding convective fits and the dashed curves are the predictions of standard ML2 convection (Böhm \& Cassinelli 1971) and are labelled by the assumed values of the mixing length ratio, $\alpha$.

\section{Discussion}

Now that we have analyzed two DBVs having different values of $T_{\text {eff }}$ we can start to see how the convective response timescale changes across the instability strip. The very large caveat here is that in order to do this we need to use published values of $T_{\text {eff }}$ and, to a lesser extent, $\log g$ for these stars. Even so, we can make progress.

First, we note that GD 358 has a larger value of $\tau_{0}$ than PG $1351+489$, so it must have a thicker convection zone and therefore be cooler. Due to the possible presence of unseen hydrogen, the spectroscopic $T_{\text {eff }}$ determination for PG $1351+489$ can be anywhere from $22600 \mathrm{~K}$ (with hydrogen) to $26100 \mathrm{~K}$ (without hydrogen; Beauchamp 1999). We indicate the position of the cooler solution with the open circle in Figure 5. Clearly, only the hotter solution for this star makes sense. Furthermore, given the slopes (values of $N$ ) determined for these stars, we see that the hottest possible solution for PG $1351+489$ is 
preferred, i.e., that with no hydrogen. This demonstrates the power of this analysis in terms of constraining $T_{\text {eff }}$.

Second, we see that the locations of these stars are broadly consistent, i.e., we can nearly draw a line connecting the two points having the appropriate slopes. Finally, we can compare these locations with the predictions of simple mixing length theory. As shown in Figure 5, we find $\mathrm{ML2} / \alpha=1.1$ (Böhm \& Cassinelli 1971) to be in reasonable agreement with these results.

Acknowledgments. This research was supported by the National Science Foundation under grant AST-0507639 and by a grant from the Delaware Asteroseismic Research Center.

\section{References}

Beauchamp, A., Wesemael, F., Bergeron,P., et al. 1999, ApJ, 516, 887

Bergeron, P., Wesemael, F., \& Beauchamp, A. 1995, PASP, 107, 1047

Böhm, K. H., \& Cassinelli, J. 1971, A\&A, 12, 21

Bradley, P. A., \& Winget, D. E. 1994, ApJ, 430, 850

Brickhill, A. J. 1992, MNRAS, 259, 519

Holberg, J. B., \& Bergeron, P. 2006, AJ, 132, 1221

Metcalfe, T. S., Nather, R. E., \& Winget, D. E. 2000, ApJ, 545, 974

Montgomery, M. H. 2005, ApJ, 633, 1142

Montgomery, M. H. 2007a, in Unsolved Problems in Stellar Physics: A Conference in Honor of Douglas Gough, ed. R. J. Stancliffe, G. Houdek, R. G. Martin, \& C. A. Tout, vol. 948. AIP, 99

Montgomery, M. H. 2007b, in ASP Conference Series, ed. A. Napiwotzki \& M. R. Burleigh, 372, 635

Winget, D. E. 1982, PhD thesis, University of Rochester

Winget, D. E., Nather, R. E., Clemens, J. C., et al. 1994, ApJ, 430, 839

Winget, D. E., van Horn, H. M., Tassoul, M., et al. 1982, ApJ, 252, L65

Wu, Y. 2001, MNRAS, 323, 248

Yeates, C. M., Clemens, J. C., Thompson, S. E., \& Mullally, F. 2005, ApJ, 635, 1239 


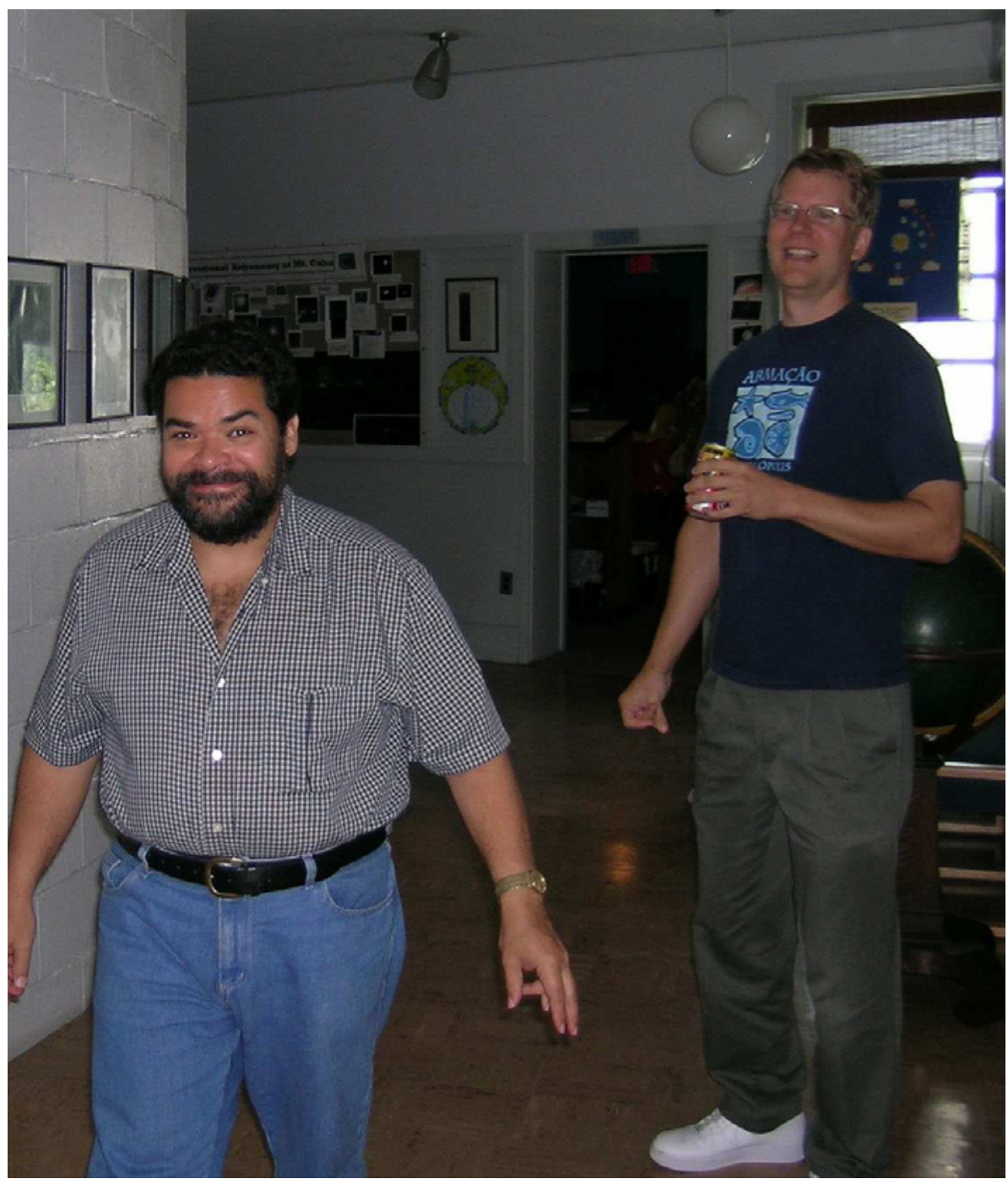

S. O. Kepler and M. H. Montgomery in Mount Cuba Observatory. 